\title{
Análisis estadístico de la percepción que tienen niñas y niños de la Enseñanza General Básica de los comportamientos violentos escolares
}

Statistical analysis of the perception that children have in Basic General Education of school violent behaviors

\author{
Volumen 17, Número 1 \\ Enero-Abril \\ pp. 1-24
}

Este número se publicó el $1^{\circ}$ de enero de 2017

DOI: http://dx.doi.org/10.15517/aie.v17i1.27199

\author{
Jenny María Artavia Granados \\ Carlomagno Araya Alpízar
}

Revista indizada en REDALYC, $\underline{\text { SCIELO }}$

Revista distribuida en las bases de datos:

\section{LATINDEX, DOAJ, REDIB, IRESIE, CLASE, DIALNET, SHERPA/ROMEO, QUALIS-CAPES, MIAR}

Revista registrada en los directorios:

ULRICH'S, $\underline{\text { REDIE}}, \underline{\text { RINACE}}, \underline{\text { OEI }}$ MAESTROTECA, PREAL, $\underline{\text { CLACSO }}$ 


\title{
Análisis estadístico de la percepción que tienen niñas y niños de la Enseñanza General Básica de los comportamientos violentos escolares \\ Statistical analysis of the perception that children have in Basic General Education of school violent behaviors
}

\author{
Jenny María Artavia Granados ${ }^{1}$ \\ Carlomagno Araya Alpízar²
}

\begin{abstract}
Resumen: Este artículo es producto de una investigación de índole mixta, realizada en el 2015, que procuró analizar si en los juegos e interacciones sociales desarrolladas durante los recreos por el estudiantado de I y II ciclo de la Enseñanza General Básica de una escuela de la Dirección Regional de Educación de Occidente (Costa Rica), existen comportamientos violentos. En el desarrollo de este estudio, se pretendió identificar el grado de percepción que posee esta población y realizar un análisis comparativo de la percepción de violencia que estos poseen, considerando las variables de sexo y nivel escolar. Como parte de los resultados, se encontró que no existen diferencias estadísticamente significativas entre los promedios del índice de violencia (IDV) percibida por las niñas y los niños. El alumnado de sexto año es el que tiene un menor promedio en el índice, esto podría indicar que a mayor edad, se tiende a aceptar comportamientos o acciones violentas como válidas, o considerar que son acciones "normales", "no agresivas". Otra situ ación observada en el estudio es que el alumnado de tercer y cuarto año presenta el promedio del índice de violencia mayor $(9,1)$, esto implica que esta población detecta con más claridad cuando una acción de sus pares es violenta.
\end{abstract}

Palabras clave: educación primaria, recreo, violencia escolar, percepción.

\begin{abstract}
This article is product of a mixed research done in 2015, it aimed to examine whether in games and social interactions that take place during the school recess, students of I and II cycle of the General Basic Education in a school of the Western Regional Bureau of Education, there are violent behaviors. In the development of this study, it was intended to identify the degree of perception of the subjects and then conduct a comparative analysis of the perception of the violence that they have, from sex and school variables. As part of the results of the study, it was found that there are no statistically significant differences among the averages of rate of violence perceived by girls and boys. In addition, sixth grade students are those who have a lower average rate, this could indicate that the older they become, they tend to accept behaviors or violent actions as valid or to consider that they are "normal", "non-aggressive" actions. Another situation observed in the study is that children of third and fourth grades have the highest average of rate of violence (9.1), this implies that they are the ones that detect, with greater clarity, when a classmate action is violent.
\end{abstract}

Keywords: primary education, school recess, school violence, perception.

\footnotetext{
1 Docente de la Sede de Occidente de la Universidad de Costa Rica.

Dirección electrónica: jargra@gmail.com

2 Profesor la Sede de Occidente, Universidad de Costa Rica. Dirección electrónica: carlo.araya@ucr.ac.cr
}

Artículo recibido: 27 de mayo, 2016

Enviado a corrección: $1^{\circ}$ de setiembre, 2016

Aprobado: 21 de noviembre, 2016 


\section{Introducción}

La escuela es un ente social muy importante que facilita el desarrollo integral de cada uno de los ciudadanos, a través de procesos educativos que permiten su formación personal, social y ética, esto con la finalidad de que estos puedan insertarse y adaptarse en el desarrollo social, cultural y económico, tanto nacional como internacional. La escuela cumple un papel medular en los procesos en los cuales se procura que los individuos se adapten a los comportamientos y exigencias de la sociedad. Desde esta perspectiva, la casa de enseñanza desempeña la principal función social de preparar a los ciudadanos, en pro de conseguir estas metas.

Una de las finalidades de la educación es justamente proporcionar a las personas las herramientas precisas para afrontar el futuro, a partir de los cambios sociales, económicos y tecnológicos que se van suscitando. Para esto debe procurar formar integralmente al estudiante de manera que se le prepare para ser un ciudadano responsable, consciente de sus deberes y derechos, capaz de relacionarse con los otros, dispuesto a desarrollar cualidades, actitudes y conductas que le permitan interrelacionarse con los demás.

El proceso de convivencia escolar resulta ser fundamental para la configuración de procesos relacionados con los valores, actitudes, sentimientos y roles que se asumirán en la comunidad. Comellas y Lojo (2009), indican que la escuela es un elemento clave en el fomento de las relaciones interpersonales que posibilitan el crecimiento humano de esa persona, "con el fin de formar personas responsables, autónomas, solidarias y críticas con la sociedad" (p. 56).

Sin embargo, es fundamental tomar en cuenta que esta institución resulta ser un ente donde se conjugan factores externos provenientes de la sociedad como son los elementos sociales, económicos, culturales, políticos y familiares; con aquellos factores internos que se generan en la institución educativa, producto de las interrelaciones personales que se suscitan entre los diferentes elementos generadores de currículo: personal docente y administrativo, estudiantes y padres de familia, que en el intercambio cotidiano y en la lucha de poderes que se genera entre los mismos, se provocan acciones de violencia.

En el medio escolar se tiende a propiciar violencia, tanto de forma explícita como oculta, violencia que ingresa del contexto social y se genera al interior de las instituciones educativas. Autores como: Abad (2002), Torrego (2006), Martínez-Otero (2005), Abramovay (2005), entre otros, señalan que la escuela es un entidad que reproduce la violencia que se suscita en el contexto social al cual sirve, pero que también es productora de violencia. 
Desde esta perspectiva, los mismos autores citan algunos elementos que a lo interno de la institución educativa, promueven comportamientos violentos, entre estos señalan: abuso y desigualdad de poder entre estudiantes, docentes y personal administrativo, estilos de enseñanza donde el proceso de enseñanza y aprendizaje se torna rígido, pasivo, mecánico y poco significativo para el estudiante, problemas de comunicación y de relaciones interpersonales entre estudiantes, personal docente y administrativo, incapacidad de trabajo en equipo, desmotivación del estudiante a partir de los bajos logros académicos, la cantidad excesiva de estudiantes por aula lo cual provoca que el docente no pueda atender sus individualidades, la reducción de espacios físicos: aulas y zonas de recreo, exceso de labores para el personal docente y las normas y reglamentos que rigen las instituciones educativas las cuales enfatizan en el empleo de modelos punitivos, los cuales establecen una sanción ante una acción tipificada por la escuela, en las normas de convivencia, como una falta, esta hace incapié en el empleo del castigo sobre la falta cometida, no permite impulsar procesos de prevención de la violencia y de convivencia pacífica.

En los últimos años, resulta alarmante el aumento de la violencia que se desarrolla en el interior de las instituciones educativas; estas cifras se logran visualizar a través de investigaciones realizadas por instituciones estatales y privadas, así como las noticias que presentan los diferentes medios de comunicación, las cuales permiten a la sociedad costarricense comprender que el fenómeno de la violencia escolar es un hecho, que está presente en el proceso educativo cotidiano desarrollado en escuelas y colegios. El fenómeno de la violencia en sus diferentes manifestaciones: física, verbal, psicológica y de maltrato o acoso escolar han ido en aumento en las instituciones, al respecto Cabezas y Monge (2013, p. 16) expresan que en la escuela costarricense:

El acoso escolar es un fenómeno que va en aumento, como se percibe a través de los diferentes estudios realizados, por lo que, no solo debe verse como un fenómeno generado entre el alumnado, sino como una situación integral que involucra a la familia, a la escuela y a una sociedad, problema que, a su vez, tiene graves consecuencias en el desarrollo socio emocional que, además, incide en la salud de la población involucrada.

A nivel internacional y nacional, los noticieros de la radio y la televisión, así como la prensa escrita, previenen diariamente a la ciudadanía de los hechos de violencia que día tras día se presentan en escuelas, colegios y universidades. Las y los ciudadanos de diferentes 
países, hemos sido alertados de la gravedad de los hechos de violencia escolar, que se suscitan entre estudiantes en las instituciones educativas. En los últimos años, los hallazgos en cuanto a violencia escolar, dejan ver acciones que van desde encontrar armas de fuego y armas blancas en los bultos, el lanzamiento de bombas de reacción química, peleas con graves heridas, como consecuencia, la muerte de un estudiante o de la directora de una escuela privada.

Hoy las escuelas se han convertido en una instancia donde estudiantes, docentes, personal administrativo y padres de familia se sienten, en muchas ocasiones, inseguros, desprotegidos y preocupados por el aumento del fenómeno de la violencia. En el caso específico del estudiantado que enfrenta cotidianamente el problema de los comportamientos violentos por parte de sus compañeros y compañeras en el ambiente escolar, percibe que la escuela no es un lugar tan seguro; al respecto Carrozo, Ponce y Morales (2014, p. 5) manifiestan que: “los niños y los jóvenes se sienten incapaces de hacer frente a los matones en la escuela y se atemorizan ante el clima de inseguridad que reina en la escuela, se hipersensibilidad frente a las presiones que le impone su entorno familiar y escolar".

\section{Comportamientos violentos que se suscitan en la escuela}

Como ya se ha hecho referencia, en el interior de las instituciones educativas se desarrollan comportamientos violentos que están relacionados con factores externos o ajenos al sistema educativo, así como aquellos que se desenvuelven en el interior de las escuelas y colegios. En el contexto interno de la escuela, se suscitan una serie de comportamientos violentos que se producen tanto de forma explícita o evidente, como de forma implícita u oculta.

Las manifestaciones de violencia explícita, son fácilmente identificables, entre estas se pueden citar:

- Violencia física la cual se identifica a través de golpes, empujones, zancadillas, entre otros. Este tipo se lleva a cabo de forma directa, se da de forma personal y se emplea la fuerza física para resolver las diferencias que se presentan. El agresor actúa directamente sobre la víctima a través de golpes físicos o sobre sus pertenencias.

- Violencia verbal, en la cual se emplean de palabras soeces y apodos, que procuran insultar, humillar, avergonzar, rechazar y ofender al compañero o compañera. Este tipo de comportamiento violento, es menos perceptible ante terceras personas, pues 
emplea expresiones orales que ofenden o humillan a la víctima, a partir de ciertos rasgos físicos, color de la piel o clase social de donde procede la víctima. Este tipo de violencia causa en la víctima la pérdida de autoestima, inseguridad, pérdida de confianza, entre otras.

- Disrupción que se presenta cuando se suscitan interferencias o comportamientos incorrectos por parte del estudiantado, esto impide a los docentes desarrollar normalmente las lecciones. Este tipo de actividades favorecen un clima de indisciplina y desorden en el salón de clases.

- Indisciplina esta se reconoce a través de los conflictos producidos entre un estudiante y su docente. En el desarrollo de este tipo de comportamientos, se percibe un conflicto en la relación educando y educador. Los actos de indisciplina en el aula, son generados por un infractor que procura imponer su voluntad sobre los demás, incluyendo al docente. Por tanto, este genera comportamientos violentos que transgreden las normas establecidas por la institución escolar, con la finalidad de causar desorden y caos, y lograr así dificultar la convivencia y el proceso de enseñanza y aprendizaje.

- El vandalismo como comportamiento violento con el cual se procura causar daños a la infraestructura y recursos de la institución educativa. Con este, el estudiante o grupo de estudiantes procuran apoderarse de los recursos físicos de la institución, así como destrozar el mobiliario e incluso dañar aulas, baños u otras áreas.

- Violencia estudiantil interinstitucional donde se presentan enfrentamientos entre estudiantes de diferentes centros educativos. Estos pueden causar serios problemas de violencia entre estudiantes.

Con respecto a la violencia de índole oculta, esta se lleva a cabo de forma encubierta y por tanto, no es fácil de ser identificada por el adulto. Entre las manifestaciones de este tipo de violencia, se encuentran:

- La violencia psicológica en la cual se producen comportamientos como: hostilidad verbal, el rechazo, la exclusión, la discriminación, entre otros. En esta la víctima recibe continuos insultos, burlas, desprecios, críticas y/o amenazas por parte del agresor. Al respecto, la Unicef (2011, p. 28), considera que "el maltrato emocional/psicológico es cualquier conducta verbal o no verbal que provoca en el niño sentimientos de 
descalificación o humillación". Este tipo de violencia puede causar en las víctimas problemas de conducta escolar y dificultades de aprendizaje.

- A través de la intimidación o bullying, la víctima es acosada y amenazada constantemente por el victimario. Este tipo de comportamientos violentos, van desde las simples ofensas, la exclusión social hasta la violencia física que ocurre de forma reiterada y permanente. Con este tipo de maltrato entre compañeros y compañeras, se realizan comportamientos violentos que pretenden insultar, ridiculizar o difundir rumores del otro.

En la intimidación se logra percibir tres sujetos: el victimario descrito como una persona violenta y fuerte, -la víctima-, caracterizada por ser sumisa y débil, la cual recibe del victimario acciones de abuso de poder de manera sistemática. $\mathrm{Y}$, por último, se identifican los espectadores de la violencia, quienes resultan ser aquellos niños o niñas compañeros de la víctima, que son conocedores de las agresiones hacia la víctima y no lo apoyan o ayudan, sino que por el contrario, se convierten o cómplices del agresor y muchas veces participan de la agresión. Para Cerezo (2007, p. 47) el bullying es "una forma de maltrato, normalmente intencionada y perjudicial, de un estudiante hacia otro compañero, generalmente más débil, al que convierte en su víctima habitual; suele ser persistente, puede durar semanas, meses e incluso años"

- También se promueven comportamientos violentos entre estudiantes relacionados con el acoso o abuso sexual. Este tipo de violencia se lleva a cabo de forma solapada, oculta y las víctimas generalmente son las mujeres. Desde esta perspectiva, se podrían identificar algunas acciones que expresan comportamientos violentos de índole sexual, entre ellas se citan: insultos y miradas obscenas, tocamientos, comentarios sexuales, estrujar con intenciones sexuales, entre otros.

\section{Metodología}

La metodología empleada en la investigación que dio origen a este artículo, es de tipo mixta, sin embargo, los datos empleados en este, corresponden a la parte cuantitativa de tipo explicativa. Hernández, et al. (2007, p. 108) manifiestan que los estudios explicativos "están dirigidos a responder por las causas de los eventos y fenómenos físicos o sociales. Su interés se centra en explicar por qué ocurre un fenómeno y en qué condiciones se manifiesta, o porqué se relacionan dos o más variables". Con este estudio se procuró identificar el grado de percepción según el sexo y el nivel escolar, que tienen niños y niñas 
de la enseñanza general básica, acerca de los comportamientos violentos que se suscitan en la escuela en estudio.

La escuela elegida para el desarrollo de este estudio, se localiza en el cantón central de San Ramón de Alajuela, Costa Rica. Esta es una institución educativa pública que forma parte del circuito 01 de la región educativa de occidente. La población que formó parte del estudio, estuvo conformada por 472 estudiantes de I y II ciclo de la enseñanza general básica.

La técnica empleada para la recolección de los datos fue el cuestionario, instrumento conformado por preguntas de tipo cerradas. Estas procuraron obtener información acerca de la percepción que tienen niños y niñas de los tipos de comportamientos violentos, que se propician en los juegos e interacciones sociales desarrolladas en los recreos entre estudiantes del I y II ciclo de la enseñanza general básica. Para lograr este objetivo, se confeccionó un instrumento en el cual se presentó a las niñas y los niños treinta situaciones o acciones que podrían suceder durante el desarrollo de los recreos. En estas se anotaron actividades en las que se suscitaban acciones violentas, así como otras no violentas. Con respecto a las situaciones en las cuales se evidenciaron acciones violentas, se presentaron algunos ejemplos donde en los juegos hubo golpes, empujones, zancadillas y se lograban escuchar apodos, se ofendía y humillaba, o se aislaba o rechazaba a algún compañero o compañera. En el apartado del análisis de la información, se presentan los datos encontrados en el estudio.

Asimismo, se presentaron algunas aseveraciones o acciones en las cuales no se evidenciaban comportamientos violentos, entre ellas se citan:

- Cuando sales a jugar con los compañeros o compañeras.

- Cuando compartes la merienda con tus compañeros o compañeras.

- Si asistes al gimnasio a jugar con los compañeros o compañeras.

- Cuando ayudas a un compañero o compañera que ha tenido un accidente en el recreo.

- Cuando acompañas a un compañero o compañera que está solo o sola, porque nadie quiere jugar con él o ella.

- Cuando vas a la soda con un compañero o compañera a comprar algún alimento.

- Al conversar con los compañeros o compañeras.

- Cuando buscas a la maestra para decirle que algún niños o niña, está peleando con otro u otra. 
- Cuando compartes juegos con tus compañeros o compañeras como brincar la cuerda, jugar bola, correr.

- Cuando conversas o juegas con la maestra.

- Cuando gritas, caminas o corres con los compañeros o compañeras por los pasillos.

- Cuando felicitas a un compañero o compañera por ganar en alguno de los juegos.

- Cuando ayudas a un compañero compañera que es amenazado o amenazada por otro.

Es importante mencionar que los resultados obtenidos de estas afirmaciones, revelaron que la población estudiantil considera en un 100\%, que en estas acciones no existen comportamientos violentos.

Con esta información, se realizó un análisis estadístico univariado y comparativo de la percepción que tienen niños y niñas de los comportamientos violentos escolares, según sexo y nivel escolar de las niñas y los niños sujetos del estudio.

El cuestionario se aplicó a los grupos en estudio, de la siguiente manera:

- Las niñas y los niños de I grado fueron llevados a la biblioteca en subgrupos de cinco estudiantes, una vez ubicados en las mesas previstas para este proceso, la investigadora dio las instrucciones de cómo se iba a contestar el cuestionario. La investigadora fue leyendo cada pregunta del cuestionario a cada subgrupo de niñas y niños. Se les dio el tiempo que requerían, se contestó las dudas y en algunos casos, se les ayudó a contestar aquellas preguntas que requerían una palabra o una oración.

- Con la niñez de II y III año el proceso se llevó a cabo de forma diferente, la investigadora solicitó al personal docente entre 20 y 25 minutos para visitar las aulas y leer a las niñas y los niños el cuestionario, para que de forma guiada, estos fueran contestando las preguntas. Durante este lapso de tiempo, se resolvieron las dudas presentadas.

- Por último, al grupo de IV, V y VI año se les leyó el cuestionario y se les dio un tiempo aproximado de 20 ó 25 minutos para que lo contestaran. También se abordaron las dudas.

\section{Análisis de los resultados}

En total se aplicó el instrumento a una muestra de 472 estudiantes, con proporciones muy semejantes entre de niñas y niños por grados. La consistencia del instrumento de 
medición de la violencia interna basado en el alfa de Cronbach es 0,813. En este sentido, un valor de 0,8 o más se considera adecuado en la investigación básica.

Es importante aclarar que los resultados de este estudio no se pueden generalizar a otras instituciones, esto por cuanto, se trabajó únicamente con una escuela de la dirección regional de educación de occidente. Esta situación se podría visualizar como una limitante del estudio.

\section{Gráfico 1.}

Distribución de los niños y las niñas encuestados según sexo y año de una escuela de la dirección regional de educación occidente, 2015

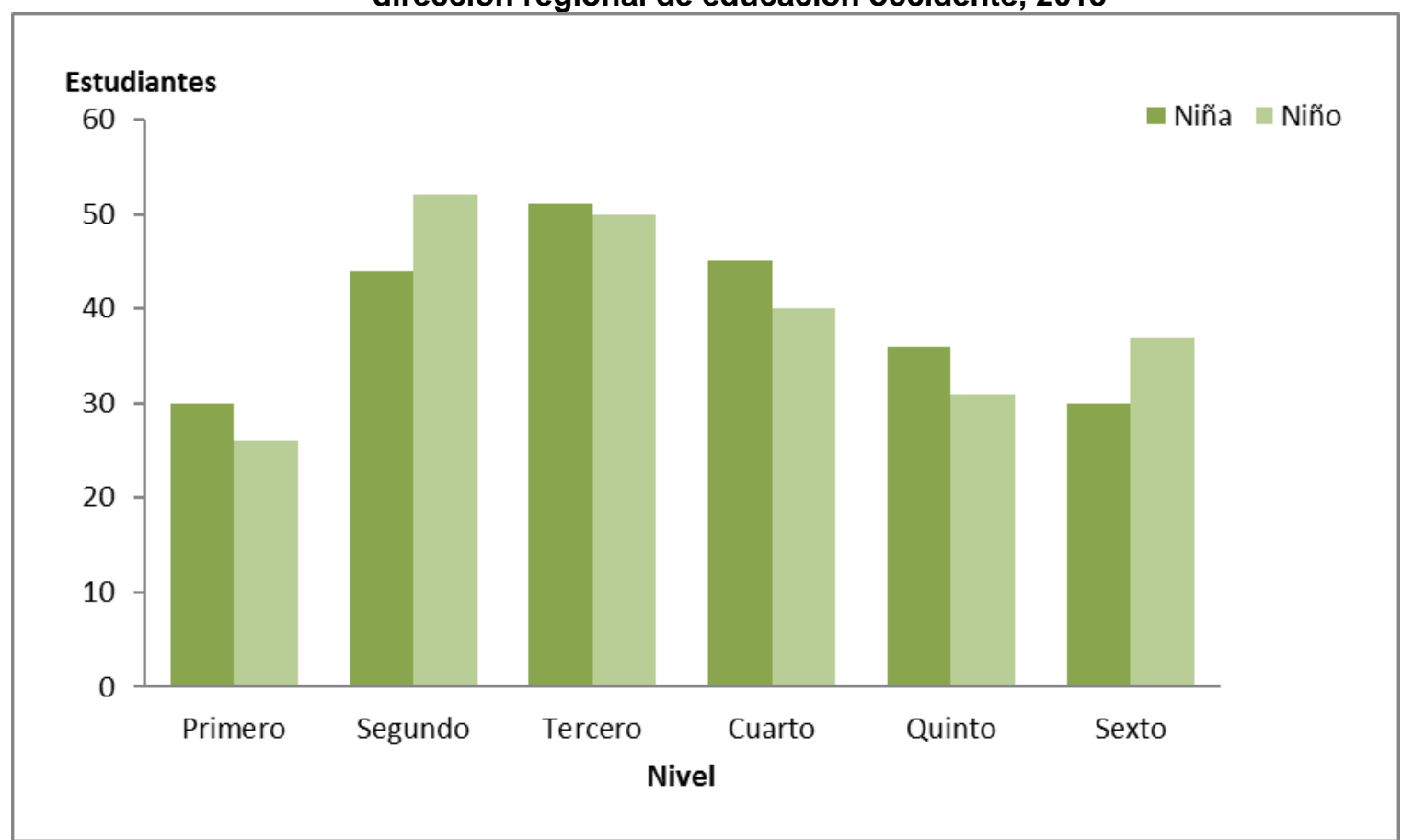

Fuente: Elaboración propia a partir de los datos obtenidos del cuestionario, aplicado a estudiantes de los grupos en estudio, 2015.

El gráfico 1 permite reconocer la distribución de los niños y las niñas según sexo y nivel educativo, que conformaron la población sujeto del estudio realizado con estudiantes de I y II ciclo de la enseñanza general básica, de una escuela localizada en la dirección regional de educación occidente. Como puede observarse, el cuestionario aplicado tomó en cuenta estudiantes de los seis niveles escolares, distribuidos de la siguiente manera:

- I año 56 estudiantes ( 30 niñas y 26 niños)

- II año 96 estudiantes (44 niñas y 52 niños)

- III año 101 estudiantes (51 niñas y 50 niños)

- IV año 85 estudiantes (45 niñas y 40 varones) 
- V año 67 estudiantes (36 niñas y 31 varones)

- VI año 67 estudiantes (30 niñas y 37 varones)

Para iniciar el análisis de los datos, se considera preciso indicar al lector la forma en que se presenta la información. En primera instancia, se realizó una agrupación de las situaciones o acciones presentadas al grupo de estudiantes, para su posterior análisis. Esta clasificación tomó en cuenta los tipos de manifestaciones de violencia: física, verbal, acoso sexual, psicológica y maltrato o bullying.

Según un estudio realizado por Cabezas y Monge (2013), existen tres formas de maltrato entre iguales que se presentan con mayor frecuencia. Al respecto las investigadoras indican que:

En primer lugar, se ubican aquellas que conllevan al maltrato físico, dentro de las que pueden citarse: los puñetazos, las patadas y jalar el cabello. En segundo lugar, están las burlas, destacándose los insultos, los apodos, los gritos y, por último, las amenazas con un factor agregado que es el uso de arma de fuego y arma blanca. Todos estos factores llevan a las víctimas al aislamiento y al retraimiento social. (2013, p. 11)

Sin embargo, en el diario convivir entre estudiantes se podría llegar a percibir que muchos de los comportamientos violentos que se suscitan entre estos, podrían llegar a visualizarse como una forma habitual de comportamiento relacional entre estudiantes. Al respecto Tomasini, Domínguez y Peralta señalan que:

En el trascurrir de la cotidianeidad escolar las y los estudiantes pueden tejer relaciones significativas de complicidad, alianzas más o menos situacionales, solidaridades, amistades, seducciones así como pueden experimentar incomodidad, malestar, humillación, etc. Se trata de un espacio social donde la relación con el otro puede asumir diversas formas. (2013, p. 4)

El proceso de socialización conlleva una serie de prácticas en las cuales se producen interrelaciones entre niños y niñas, quienes procuran alcanzar procesos donde se reconocen y son reconocidos. Desde esta perspectiva, se inicia uno donde cada persona se muestra desde diferentes ámbitos con otra. A nivel escolar las relaciones interpersonales suelen estar supeditadas a la aceptación del otro, aunque esto implique que se deban tolerar o aceptar ciertos comportamientos que podrían incluso caer en diversas conductas violentas. 
Precisamente, el estudio realizado procuró identificar si la población estudiantil tiene la capacidad de percibir cuándo ha recibido comportamientos violentos físicos, verbales, de acoso sexual, psicológicos y de maltrato o intimidación, por parte de sus compañeros 0 compañeras.

En el cuadro 1 se observa las acciones que se podrían suscitar entre estudiantes durante el desarrollo del recreo, y que podrían manifestar comportamientos violentos de índole físico. En las primeras tres situaciones escolares, que se presentaron a las niñas y a los niños:

1) Estás jugando con tus compañeros o compañeras y de pronto uno de ellos comienza a golpearte.

2) Vas corriendo y un niño te pega una zancadilla.

3) Estás jugando y de pronto un niño o niña te empuja.

Los resultados observados, permiten afirmar que la violencia física resulta generalmente fácil de percibir por el estudiantado en todas sus manifestaciones. Las situaciones presentadas a las niñas y a los niños, están ligadas a comportamientos de violencia física de tipo directa, los cuales son totalmente visibles y pueden involucrar acciones como: el golpe, la patada, el empujón, el mordisco, el pellizco, entre otras.

Generalmente esta conducta es observada por el otro, como una "verdadera" conducta violenta. Se hace referencia a esta expresión porque entre los menores e inclusive entre las personas adultas, es común escuchar que el comportamiento en el cual uno pega a otro es aquel donde se lleva a cabo un acto de violencia. No así cuando niños y niñas se agreden verbal o psicológicamente entre estos.

Cuadro 1. San Ramón: Opinión del estudiantado de I y II ciclo, acerca de si las situaciones planteadas pueden considerarse como comportamientos violentos de índole física, 2015 (en porcentajes)

\begin{tabular}{l|c|c|c|c|c|c}
\hline Situaciones escolares & Primero & Segundo & Tercero & Cuarto & Quinto & Sexto \\
\hline $\begin{array}{l}\text { Estás jugando con tus compañeros (as) y de } \\
\text { pronto uno de ellos, comienza a golpearte }\end{array}$ & 96 & 96 & 95 & 96 & 96 & 89 \\
\hline $\begin{array}{l}\text { Si vas corriendo y un niño te pega una } \\
\text { zancadilla }\end{array}$ & 96 & 95 & 96 & 94 & 90 & 90 \\
\hline $\begin{array}{l}\text { Estas jugando y de pronto un niño (a) te } \\
\text { empuja. }\end{array}$ & 95 & 95 & 93 & 93 & 96 & 88 \\
\hline
\end{tabular}

Fuente: Elaboración propia a partir de los resultados obtenidos de los cuestionarios aplicados al estudiantado de la escuela en estudio. 
Los resultados presentados en el cuadro anterior, permiten observar que los porcentajes de estudiantes que reconocen estas situaciones como violentas, son cercanos al $90 \%$ o superiores, las cuales se pueden considerar estadísticamente altas.

La violencia verbal es aquella en la cual el victimario a través del lenguaje verbal ofende, humilla o avergüenza a su víctima. Según Machado y Guerra (2009), la violencia verbal es el tipo de violencia más empleada por los estudiantes. En un estudio realizado por Chagas (2005), la investigadora manifiesta que:

Según los profesores, la violencia verbal es la faceta más "común y natural" del trato entre los niños y la que se considera más inocua, aunque esto no corresponda siempre a la realidad, ya que el daño de la devaluación o la humillación puede ocasionar graves lesiones en la autoestima y, como consecuencia, en los vínculos interpersonales. (p. 1077)

Al consultar al estudiantado sobre el empleo de apodos, uso de malas palabras y de palabras que ofenden o humillan, estos consideran que sí existe violencia en estas acciones (Ver cuadro 2). A continuación, se procede a analizar cada una de las aseveraciones hechas a la población estudiantil.

Cuadro 2. San Ramón: Opinión del estudiantado acerca de si las situaciones planteadas puede considerarse como comportamientos violentos de tipo verbal, 2015 (en porcentajes)

\begin{tabular}{l|c|c|c|c|c|c}
\hline Situaciones Escolares & Primero & Segundo & Tercero & Cuarto & Quinto & Sexto \\
\hline Cuando los compañeros (as) te dicen apodos & 88 & 83 & 79 & 76 & 76 & 75 \\
\hline $\begin{array}{l}\text { Cuando los compañeros (as) te dicen malas } \\
\text { palabras y te hacen sentir mal }\end{array}$ & 96 & 93 & 91 & 93 & 94 & 90 \\
\hline $\begin{array}{l}\text { Algún compañero (a) le dijo a los demás, } \\
\text { alguna cosa que te ofendió, humilló o } \\
\text { avergonzó. }\end{array}$ & 82 & 95 & 90 & 87 & 94 & 84 \\
\hline
\end{tabular}

Fuente: Elaboración propia a partir de los resultados obtenidos de los cuestionarios aplicados al grupo estudiantil de la escuela en estudio.

En la situación "Cuando los compañeros(as) te dicen apodos", se observa diferencias estadísticamente significativas en los porcentajes según el grado del escolar ( $p$-valor=0,000). Obsérvese que mientras en primer grado esta situación la determinan como violentas $88 \%$, en tercero $79 \%$ y cuarto el $76 \%$ no la consideran de esta manera. Este porcentaje es aún más pequeño para el estudiantado de sexto $(75 \%)$. Pareciera que conforme se avanza en 
edad, el grupo estudiantil de II ciclo de la enseñanza general básica, no perciben que exista violencia en estas acciones, o tal vez consideran que este tipo de expresiones son "normales" entre iguales.

En la situación "Los compañeros (as) te dicen malas palabras y te hacen sentir mal", el grupo entrevistado, en un porcentaje de $90 \%$ o más según los grados que cursan, efectivamente la determinan como una manifestación de violencia escolar.

Con respecto a si "Algún compañero (a) le dijo a los demás, alguna cosa que te ofendió, humilló o avergonzó", esta es considera como una acción de violencia con mayor porcentaje para el estudiantado de segundo y quinto grado escolar. Mientras que para quienes cursan el primer y sexto grado, el porcentaje es aproximadamente $10 \%$ menor, tomando valores de $82 \%$ y $84 \%$, respectivamente.

Al analizar los porcentajes de respuestas obtenidas a partir del empleo de las malas palabras y del uso de palabras que humillan, ofenden o avergüenzan, es importante indicar que pareciera que este tipo de expresiones, son percibidas por la mayoría de niños y niñas, como acciones que maltratan más.

La violencia verbal es más imperceptible que la violencia física, pero hay en la palabra que agrede efectos que son más graves que el golpe físico. Este tipo de violencia emplea constante palabras y comentarios que tienden a degradar, insultar y humillar, aspecto que en última instancia causa un gran dolor en la víctima, al respecto Valadez indica que:

En el maltrato verbal se utiliza una comunicación agresiva, donde se expresa lo que se siente, lo que se quiere, lo que se piensa a costa de los derechos y los sentimientos de los demás, tendiendo a humillar y a atacar cunado no puede "salirse con la suya" fomentando la culpa y el resentimiento en los otros, no desarrollándose la negociación ni el diálogo en el proceso comunicativo. (2008, p. 19)

También es importante señalar que cuando se suscitan comportamientos de violencia verbal, el victimario elige aquella o aquellas palabras que sabe van a producir mayor dolor en la víctima y que van a permitir ejercer mayor dominio sobre esta, para ello astutamente emplea un cierto tono de voz y generalmente cambia el volumen de esta.

El cuadro 3 permite al lector identificar la percepción que tienen el estudiantado con respecto a los comportamientos de maltrato o intimidación (bulliyng). El maltrato escolar o acoso escolar es un tipo de violencia en la cual se presenta un abuso de poder por parte de aquellos estudiantes más fuertes físicamente o de mayor edad, contra otros más débiles o de 
menor edad. Castro (2009, p. 62) manifiesta que en el fenómeno del maltrato, la principal particularidad presentada es el "abuso de poder, de dominación y subordinación entre las personas, de tal manera que se dejan de lado los valores humanitarios, el respeto al otro". El mismo autor señala que existen diferentes tipos de agresiones de índole intimidatorio: físico, verbal y de exclusión social. Estas manifestaciones de agresión perjudican la autoestima del otro, al respecto Valadez (2008, p. 21) manifiesta que con este tipo de maltrato psicológico se causa daño en:

Las habilidades de un niño/adolescente, lesionando su autoestima, su capacidad de relacionarse y su habilidad para expresarse y sentir. Esto ocasiona un deterioro en su personalidad, socialización y en general en el desarrollo armónico de sus emociones y habilidades, limitando e inhabilitando su desarrollo potencial.

El daño psicológico causado por el maltrato escolar es sumamente difícil de asumir por el niño o la niña preadolescente, porque se encuentra ante la disyuntiva de socializar con sus pares aunque esto le cause dolor o alejarse del grupo de compañeros y compañeras para aislarse, aspecto que también le va a causar dolor y frustración. Y la otra alternativa que le queda, es soportar en silencio, el maltrato de un compañero o compañera.

A continuación se presenta la información relacionada con la percepción de la población estudiantil con respecto a aquellos comportamientos violentos que tienen que ver con el maltrato o intimidación.

A la población sujeto del estudio, se le presentaron una serie de situaciones en las cuales se describían acciones relacionadas con el maltrato entre estudiantes. Las tres primeras situaciones observadas en el cuadro 3 están totalmente ligadas al maltrato directo que desarrolla el victimario y que procuran la exclusión social de la víctima. Con respecto a las expresiones que manifiestan:

- Hay compañeros o compañeras que no te dejan hablar o jugar con otros.

- Algunos compañeros o compañeras no te dejan compartir con ellos algún juego o conversación en el recreo.

- Cuando algunos compañeros o compañeras se apartan de ti y no les gusta que juegues o salgas con ellos al recreo. 
Cuadro 3. San Ramón: Opinión del estudiantado de I y II ciclos, acerca de si las situaciones planteadas pueden considerarse como comportamientos de maltrato o intimidación, 2015 (en porcentajes)

\begin{tabular}{l|c|c|c|c|c|c}
\hline $\begin{array}{l}\text { Situaciones que se podrían suscitar } \\
\text { entre escolares }\end{array}$ & Primero & Segundo & Tercero & Cuarto & Quinto & Sexto \\
\hline $\begin{array}{l}\text { Hay compañeros (as) que no te dejan hablar } \\
\text { o jugar con otros }\end{array}$ & 80 & 80 & 84 & 73 & 82 & 78 \\
\hline $\begin{array}{l}\text { Algunos compañeros (as) no te dejan } \\
\text { compartir con ellos algún juego o } \\
\text { conversación en el recreo }\end{array}$ & 82 & 58 & 81 & 48 & 64 & 74 \\
\hline $\begin{array}{l}\text { Cuando algunos compañeros (as) se } \\
\text { apartan de ti y no les gusta que juegues o } \\
\text { salgas con ellos al recreo }\end{array}$ & 89 & 84 & 84 & 79 & 81 & 85 \\
\hline $\begin{array}{l}\text { Existen compañeros que te prohíben decir a } \\
\text { la maestra o darle quejas a la maestra } \\
\text { cuando otros te están molestando, } \\
\text { humillando, amenazando o pegando. }\end{array}$ & 89 & 90 & 92 & 93 & 88 & 94 \\
\hline $\begin{array}{l}\text { Cuando la maestra no escucha o no presta } \\
\text { atención a las quejas, cuando eres víctima } \\
\text { de que otro niño o niña te pegue, humille, } \\
\text { diga malas palabras o apodos o te } \\
\text { amenace. }\end{array}$ & 51 & 93 & 86 & 94 & 93 & 90 \\
\hline $\begin{array}{l}\text { Cuando hay niños que tocan tus partes } \\
\text { íntimas }\end{array}$ & 96 & 99 & 95 & 95 & 90 & 88 \\
\hline
\end{tabular}

Fuente: Elaboración propia a partir de los resultados obtenidos de los cuestionarios aplicados al grupo estudiantil de la escuela en estudio.

En estas se hace referencia a la exclusión social en la cual, el victimario pretende marginar a la víctima de ciertas actividades y del resto de sus compañeros o compañeras.

Al realizar el análisis estadístico de estos ítems, fue posible llegar a los siguientes resultados:

En las situaciones del "recreo donde hay compañeros o compañeras que no te dejan hablar o jugar con otros y que algunos compañeros o compañeras no te dejan compartir con ellos algún juego o conversación en el recreo", son percibidas por el estudiantado en porcentajes cercanos al 80 \% como violencia escolar. Con respecto a que "hay compañeros o compañeras que no te dejan hablar o jugar con otros", el grupo de estudiantes de cuarto año la consideran como violencia, en un $73 \%$. En los otros grados, los porcentajes son de aproximadamente el 80 \%. La situación es mucho más preocupante con respecto a la situación en donde se enuncia que "algunos compañeros (as) no te dejan compartir con ellos algún juego o conversación en el recreo", pues resulta que el estudiantado de segundo y cuarto año la consideran como una actitud violenta, solamente en un $58 \%$ y $48 \%$, respectivamente. Aunque en primer y tercer grado los porcentajes son del $82 \%$ y $81 \%$, 
siguen inferiores a lo esperado de porcentajes superiores o iguales al $90 \%$. En ambas situaciones de violencia, se determina que hay diferencias en los porcentajes estadísticas significativas ( $p$-valor $=0,000)$.

Para el grupo de primer grado la situación que se presentó y que indica que "cuando algunos compañeros (as) se apartan de ti y no les gusta que juegues o salgas con ellos al recreo", es reconocida en un 89 \% de los estudiantes como de violencia escolar. En los otros grados escolares, los porcentajes son significativamente inferiores, siendo el grupo de cuarto grado el que en menos porcentaje (79 \%) la reconocen como agresiva.

En las situaciones donde se afirma que "existen compañeros que te prohíben decir a la maestra o darle quejas a la maestra cuando otros te están molestando, humillando, amenazando o pegando", los porcentajes son en todos los grados escolares cercanos al 90 \%. Cabe recalcar que para el grupo de sexto grado, el porcentaje para la primera situación alcanza el $94 \%$.

En la situación que especifica que "cuando la maestra no escucha o no presta atención a las quejas cuando eres víctima de que otro niño o niña te pegue, humille, diga malas palabras o apodos o te amenace", las diferencias en los porcentajes por grado escolar, son significativamente diferentes ( $p$-valor $=0,000$ ). Aproximadamente la mitad del grupo de primer grado (51 \%) no considera esta situación como violencia escolar, resultando significativamente a los demás grados, especialmente, el estudiantado de cuarto grado, donde el porcentaje es $94 \%$. Para el grupo de segundo y cuarto grado alcanza un $93 \%$.

De acuerdo con las manifestaciones de acoso escolar, se preguntó si "cuando existen niños que tocan tus partes íntimas, existe violencia escolar", sus respuestas permiten evidenciar que en los niveles desde 1 y hasta 5 años, todo el grupo opina en un $90 \%$ y más, que sí existe violencia en este acto. Por tanto, existe un alto porcentaje de concordancia en la percepción, porque para la mayoría esta acción implica un comportamiento de violencia, siendo el grupo de segundo grado, que en su totalidad la consideran como agresiva (99 \%). No obstante, en el grupo de sexto grado, el porcentaje es significativamente menor (88\%), indicando que esta situación es menos calificada para este como de agresión escolar. 
Cuadro 4. San Ramón: Opinión del estudiantado de I y II ciclo sobre si las situaciones planteadas pueden considerarse como comportamientos de intimidación o matonismo, 2015 (en porcentajes)

\begin{tabular}{l|c|c|c|c|c|c}
\hline $\begin{array}{l}\text { Situaciones que se podrían suscitar entre } \\
\text { escolares }\end{array}$ & Primero & Segundo & Tercero & Cuarto & Quinto & Sexto \\
\hline $\begin{array}{l}\text { Cuando un compañero exige que debes } \\
\text { darle dinero, porque si no te va a pegar. }\end{array}$ & 96 & 94 & 96 & 93 & 96 & 91 \\
\hline $\begin{array}{l}\text { Existen compañeros (as) que te amenazan } \\
\text { que te van a pegar. }\end{array}$ & 91 & 96 & 88 & 96 & 91 & 91 \\
\hline $\begin{array}{l}\text { Cuando alguien te quita la merienda o alguno } \\
\text { de los útiles escolares (lápiz, borrador, etc.) }\end{array}$ & 80 & 90 & 79 & 92 & 87 & 82 \\
\hline
\end{tabular}

Fuente: Elaboración propia a partir de los resultados obtenidos de los cuestionarios aplicados al grupo estudiantil de la escuela en estudio.

El cuadro 4 permite reconocer otros tipos de comportamientos violentos que están ligados con el matonismo, que se puede suscitar entre estudiantes. Independientemente del nivel donde se ubica el estudiantado, el porcentaje que reconoce la situación de violencia "cuando un compañero exige que deba darle dinero porque si no le va a pegar", resulta superior al $90 \%$. De igual manera, en la situación "existen compañeros (as) que amenazan que le van a pegar", los porcentajes por grado son superiores también a $90 \%$, con excepción en tercer grado donde este porcentaje es menor en 2 puntos, por ende alcanzando el $88 \%$.

El estudiantado de cuarto grado, reconocen con mayor porcentaje (92\%), la situación cuando alguien te quita la merienda o alguno de los útiles (lápiz, borrador, etc.) como violencia escolar. Siendo el grupo de tercer grado el que menos perciben la situación señalada anteriormente como violenta $(79 \%)$. Estas diferencias en los porcentajes son estadísticamente significativas ( $p$-valor $=0,000$ ). Se parte de la hipótesis de que los porcentajes son iguales en todos los grados.

Con el propósito de resumir las 15 posibles situaciones escolares analizadas de forma individual representadas en los cuadros antes presentados, se construyó un índice de violencia escolar. Para cada situación, si la respuesta proporcionada por la estudiante o el estudiante era la correcta, se asignó una ponderación de 3 puntos en caso contrario, cuando la respuesta es incorrecta, no se dio ningún puntaje. Además, el rango de variación del índice se transformó para una escala de 0 hasta 10, donde el cero es el puntaje mínimo que indica la peor situación para un estudiante o una estudiante, y el puntaje 10 para el estudiante o la estudiante que tiene una visión perfecta de cuáles situaciones son las que caracterizan la violencia escolar. 
Los resultados del estudio mostraron que no hay diferencias estadísticamente significativas entre los promedios aritméticos del índice de violencia (IDV) entre niñas y niños. La media aritmética de las niñas es 9,0 y los niños presentaron un promedio de 8,9.

Las niñas y niños de tercer y cuarto año presentan el promedio del índice de violencia mayor $(9,1)$, esto implica, que son ellos lo que detectan con mayor claridad, cuando una acción de sus compañeros o compañeras, resulta ser violenta. El estudiantado de primero y quinto año tiene igual promedio aritmético (8,8 puntos). En tanto, el grupo de sexto año es el que tiene un menor promedio en el índice esto podría indicar que a mayor edad, se tiende aceptar comportamientos o acciones violentas como válidas, o considerar que son acciones "normales", "no agresivas". Debido a lo anterior, el promedio aritmético del índice de violencia resulta significativamente diferentes según años a un nivel de confianza del $90 \%$ ( $p$-valor= 0,075).

Mediante el cuadro 5, se puede observar que el promedio del índice de violencia por nivel escolar, para el estudiantado de sexto año resultan significativamente diferentes con respecto a los escolares de segundo ( $p$-valor $=0,027)$, tercer ( $p$-valor $=0,012)$ y cuarto $(p$ valor $=0,017)$. En las otras posibles comparaciones por nivel, no se encontraron diferencias estadísticamente significativas.

Por una parte, al considerar el sexo de los estudiantes de I y II ciclo de la enseñanza general básica, solamente existen diferencias estadísticamente significativas entre los promedios aritméticos en el índice de violencia de los niños y las niñas de primer año, mientras los niños tienen una media de 8,5, las niñas obtienen una media de 9,1 significativamente mayor a los niños (obsérvese el cuadro número 6). En general, aunque las niñas tienen puntajes menores en el índice de violencia que los niños, las diferencias en los promedios aritméticos no son estadísticamente significativas, con excepción del caso citado, entre niños y niñas de primer año.

Por otra parte, la violencia escolar se puede subdividir en diferentes tipos o categorías, concretamente física, verbal, psicológica (rechazo y aislamiento), maltrato (intimidación o matonismo) y violencia sexual. En este sentido, en la violencia verbal se encontraron diferencias significativas entre niños y niñas ( $p$-valor=0,016). La media aritmética en violencia verbal para los niños es 9,0 en tanto, las niñas presentan un valor promedio inferior de 8,4. En los otros tipos de violencia no hay diferencias estadísticamente significativas entre las niñas y los niños. 
Cuadro 5. Comparaciones pareadas del índice de violencia escolar según grado de escolaridad.

\begin{tabular}{|c|c|c|c|c|c|c|}
\hline \multirow[b]{2}{*}{ (I) Nivel } & \multirow[b]{2}{*}{ (J) Nivel } & \multirow[b]{2}{*}{$\begin{array}{c}\text { Diferencia de } \\
\text { medias }(1-J)\end{array}$} & \multirow[b]{2}{*}{ Error estándar } & \multirow[b]{2}{*}{ Sig. } & \multicolumn{2}{|c|}{ Intervalo de confianza al $95 \%$} \\
\hline & & & & & Límite inferior & Límite superior \\
\hline \multirow[t]{5}{*}{ Primero } & Segundo & -.1587 & .41067 & .699 & -.9657 & .6483 \\
\hline & Tercero & .1892 & .40691 & .642 & -.6104 & .9888 \\
\hline & Cuarto & .3200 & .42034 & .447 & -.5060 & 1,1461 \\
\hline & Quinto & .0631 & .44220 & .887 & -.8059 & 9321 \\
\hline & Sexto & .8103 & .44220 & .168 & -.2586 & 1,4793 \\
\hline \multirow[t]{5}{*}{ Segundo } & Primero & .1587 & .41067 & .699 & -.6483 & .9657 \\
\hline & Tercero & .3479 & .34813 & .318 & -.3362 & 1,0320 \\
\hline & Cuarto & .4788 & .36374 & .189 & -.2360 & 1,1936 \\
\hline & Quinto & .2218 & .38879 & .569 & -.5422 & 9858 \\
\hline & Sexto & $.7691^{\circ}$ & .38879 & .049 & .0050 & 1,5331 \\
\hline \multirow[t]{5}{*}{ Tercero } & Primero &,- 1892 & .40691 & .642 & -.9888 & .6104 \\
\hline & Segundo & -.3479 & .34813 & .318 & $-1,0320$ & .3362 \\
\hline & Cuarto & .1308 & .35949 & .716 & -.5756 & .8373 \\
\hline & Quinto &.,- 1261 & .38482 & .743 & -.8823 & .6301 \\
\hline & Sexto & .4212 & .38482 & .274 & -.3351 & 1,1774 \\
\hline \multirow[t]{5}{*}{ Cuarto } & Primero & -.3200 & .42034 & .447 & $-1,1461$ & .5060 \\
\hline & Segundo & -.4788 & .38374 & .189 & $-1,1936$ & .2360 \\
\hline & Tercero & -.1308 & .35949 & .716 & -.8373 & .5756 \\
\hline & Quinto & -.2570 & .39900 & .520 & $-1,0410$ & .5271 \\
\hline & Sexto & .2903 & .39900 & .467 & -.4938 & 1,0744 \\
\hline \multirow[t]{5}{*}{ Quinto } & Primero & -.0631 & .44220 & .887 & -.9321 & .8059 \\
\hline & Segundo & -.2218 & .38879 & .569 & -.9858 & .5422 \\
\hline & Tercero & .1261 & .38482 & .743 & -.6301 & .8823 \\
\hline & Cuarto & .2570 & .39900 & .520 & -.5271 & 1,0410 \\
\hline & Sexto & .5473 & .42197 & .185 & -.2820 & 1,3765 \\
\hline \multirow[t]{5}{*}{ Sexto } & Primero & -.6103 & .44220 & .168 & $-1,4793$ & .2586 \\
\hline & Segundo & $-7691^{\circ}$ & .38879 & .049 & $-1,5331$ & -.0050 \\
\hline & Tercero & -.4212 & .38482 & .274 & $-1,1774$ & .3351 \\
\hline & Cuarto & -.2903 & .39900 & .467 & $-1,0744$ & .4938 \\
\hline & Quinto & -.5473 & .42197 & .195 & $-1,3765$ & .2820 \\
\hline
\end{tabular}

Fuente: Elaboración propia a partir de los resultados obtenidos de los cuestionarios aplicados al estudiantado de la escuela en estudio. 
Cuadro 6. Análisis de variancia del índice de violencia y sus componentes según sexo del escolar.

\begin{tabular}{|c|c|c|c|c|c|c|}
\hline & & $\begin{array}{l}\text { Suma de } \\
\text { cuadrados }\end{array}$ & $\mathrm{gl}$ & $\begin{array}{c}\text { Media } \\
\text { cuadrática }\end{array}$ & $\mathrm{F}$ & Sig. \\
\hline \multirow[t]{3}{*}{ Indice de violencia } & Entre grupos & 1,057 & 1 & 1,057 &, 771 & 380 \\
\hline & Dentro de grupos & 643,951 & 470 & 1,370 & & \\
\hline & Total & 645,007 & 471 & & & \\
\hline \multirow[t]{3}{*}{ Violencia Física } & Entre grupos & ,094 & 1 & ,094 & ,029 &, 865 \\
\hline & Dentro de grupos & 1533,992 & 470 & 3,264 & & \\
\hline & Total & 1534,087 & 471 & & & \\
\hline \multirow[t]{3}{*}{ Violencia verbal } & Entre grupos & 35,805 & 1 & 35,805 & 5,897 & 016 \\
\hline & Dentro de grupos & 2853,625 & 470 & 6,072 & & \\
\hline & Total & 2889,430 & 471 & & & \\
\hline \multirow[t]{3}{*}{ Rechazo y aislamiento } & Entre grupos & 10,381 & 1 & 10,381 & 1,551 &, 214 \\
\hline & Dentro de grupos & 3146,469 & 470 & 6,695 & & \\
\hline & Total & 3156,850 & 471 & & & \\
\hline \multirow[t]{3}{*}{ Intimidación o matonismo } & Entre grupos & ,305 & 1 & ,305 &, 080 & ,777 \\
\hline & Dentro de grupos & 1791,017 & 470 & 3,811 & & \\
\hline & Total & 1791,322 & 471 & & & \\
\hline \multirow[t]{3}{*}{ Violencia psicológica } & Entre grupos & 2,413 & 1 & 2,413 &, 645 & , 422 \\
\hline & Dentro de grupos & 1758,018 & 470 & 3,740 & & \\
\hline & Total & 1760,431 & 471 & & & \\
\hline \multirow[t]{3}{*}{ Maltrato sexual } & Entre grupos & 415 & 1 & ,415 & 1,925 & , 166 \\
\hline & Dentro de grupos & 101,407 & 470 &, 216 & & \\
\hline & Total & 101,822 & 471 & & & \\
\hline \multirow[t]{3}{*}{ No hay violencia } & Entre grupos & 031 & 1 & 031 & ,021 & 885 \\
\hline & Dentro de grupos & 704,842 & 470 & 1,500 & & \\
\hline & Total & 704,873 & 471 & & & \\
\hline
\end{tabular}

Fuente: Elaboración propia a partir de los resultados obtenidos de los cuestionarios aplicados al estudiantado de la escuela en estudio.

\section{Conclusiones}

Una vez analizados los datos del estudio, se logró identificar que las niñas y los niños presentan algunas diferencias con respecto a la percepción que tienen sobre los comportamientos violentos que se podrían suscitar en los juegos e interacciones sociales, que se desarrollan durante los recreos escolares. Al respecto, se indican los principales hallazgos del estudio:

- A un alto porcentaje de la población estudiantil de I y II ciclo de la enseñanza general básica (EGB), le resulta fácil percibir los comportamientos violentos de índole físico (empujones, patadas, golpes, entre otros), que provienen de sus compañeros y compañeras. 
- En cuanto a la violencia verbal, se logra identificar que para las niñas y los niños de I ciclo de EGB, el apodo resulta ser un comportamiento violento. Pero la población estudiantil de II ciclo, no percibe que en esta acción exista violencia. Por su parte, el uso de malas palabras entre estudiantes es percibido por todos los niños y todas las niñas, como un comportamiento violento. El uso de vocablos que buscan ofender, humillar o avergonzar a niños y niñas, un alto porcentaje de la población estudiantil las percibe como parte de los comportamientos violentos. Pareciera que para niños y niñas, el empleo de palabras o frases que busquen el insulto, el sometimiento o la puesta en ridículo ante otros, es totalmente observada como un comportamiento violento.

- La percepción poseída en cuanto a los comportamientos de maltrato e intimidación entre la población estudiantil, nos permite evidenciar que, en un alto porcentaje, niños y niñas logran visualizar la existencia de comportamientos violentos en aquellas acciones relacionadas con la marginación de la víctima en actividades que esta podría compartir con sus compañeros y compañeras. Sin embargo, es importante destacar que la población estudiantil de los grados superiores (IV, $\mathrm{V}$ y $\mathrm{VI}$ ) considera, en porcentajes inferiores, que se produce violencia cuando no la dejan compartir juegos o conversaciones con otros.

- Con respecto a la percepción que se posee de los comportamientos de maltrato e intimidación, se logró identificar que un alto porcentaje de estudiantes logran visualizar comportamientos violentos de exclusión social, en acciones relacionadas con la marginación de la víctima de actividades que podría compartir con sus compañeros y compañeras. Sin embargo, es importante distinguir que la población estudiantil de los grados superiores (IV, V y VI) considera en porcentajes inferiores, que se produce violencia cuando sus compañeros y compañeras, no la dejan compartir juegos o conversaciones.

- En cuanto al acoso escolar de índole sexual, en altos porcentajes niños y niñas perciben comportamientos violentos, cuando otros tocan sus partes íntimas.

- Las respuestas dadas por el estudiantado en relación con las acciones que reflejan comportamientos de intimidación o matonismo, en un alto porcentaje logran percibir que estas, resultan ser comportamientos violentos. 
- Mediante el análisis estadístico de los resultados del estudio, se puede concluir que no hay diferencias estadísticamente significativas entre los promedios del índice de violencia (IDV) entre niñas y niños.

- Los estudiantes de sexto año son los que tienen el menor promedio en el índice, esto podría indicar que a mayor edad, se tiende a aceptar comportamiento o acciones violentas como válidas o considerar que son acciones "normales", "no agresivas".

- Otra situación observada en el estudio, es que las niñas y los niños de tercer y cuarto nivel presentan el promedio del índice de violencia mayor $(9,1)$, esto implica que son quienes detectan con mayor claridad cuando una acción de sus pares es violenta.

- Con respecto a los componentes de la violencia escolar, solamente en la violencia verbal se encontró una diferencia estadísticamente significativa entre los promedios entre niños y niñas.

\section{Recomendaciones}

Una vez concluido el estudio, se considera oportuno puntualizar algunas recomendaciones:

- Reforzar en los procesos de mediación pedagógica desarrollada en los salones de clase, aquellas competencias que procuren la convivencia pacífica y la resolución de los conflictos sin violencia entre el estudiantado.

- El personal docente y administrativo debe estar vigilante ante los comportamientos violentos que se susciten entre estudiantes, para desarrollar acciones de prevención y abordaje integral de la problemática.

- Capacitar al personal docente y administrativo en la detección y abordaje de la violencia escolar en todas sus manifestaciones: física, psicológica, verbal, intimidación o bullying y acoso sexual.

- Brindar apoyo y la confianza necesaria a aquellos estudiantes que recurran al personal docente o administrativo cuando sean víctimas de comportamientos violentos en el ambiente escolar y logren romper el vínculo de la violencia.

- La institución educativa debe procurar planificar, organizar y ejecutar campañas de divulgación de información acerca de la violencia escolar, resolución de los conflictos y la convivencia pacífica. En el desarrollo de estas campañas se deben incluir a la comunidad escolar y a las familias.

- Brindar orientación y capacitación a padres y madres de familia, en temas relacionados con el fomento en sus hogares de una adecuada autoestima y habilidades sociales en 
los hijos e hijas, así como en la detección de la presencia de comportamientos violentos.

- Se considera oportuno que la temática de este estudio, sea investigada en otras instituciones educativas de la zona y del país en general, dada la importancia que tiene para la ciudadanía, obtener mayor información de la problemática de la violencia en las instituciones educativas.

\section{Referencias}

Abad, Susana. (2002). Acerca de las conductas violentas en el aula. Cuando la asimetría lleva a la humillación. Ensayos y experiencias, (44), 30-45.

Abramovay, Miriam. (2005). Violencia en las escuelas: un gran desafío: Revista Iberoamericana de Educación, (38), 13-26.

Artavia, Jenny. (2015). Comportamientos violentos en los juegos e interacciones sociales que se suscitan entre las y los estudiantes de I Ciclo de la Enseñanza General Básica, durante el desarrollo de los recreos en una escuela del circuito 01 de la Dirección Regional de Educación de Occidente (Informe Final de Investigación). San José, Costa Rica: Instituto de Investigación en Educación (INIE), Universidad de Costa Rica.

Cabezas, Hannia y Monge, Melania. (2013). Violencia escolar, un problema que aumenta en la escuela Primaria costarricense. Revista Actualidades Investigativas en Educación, 13(2), 1-20. Recuperado de http://revista.inie.ucr.ac.cr/index.php/aie/article/view/596

Carrozo, Julio César, Zapata, Luis y Morales, Luis Benitez. (2014). ¿Es la escuela un lugar seguro? Recuperado http://observatorioperu.com/2014/Novedades/Marzo/Carozzo\%20Es\%20la\%20escuela \%20un\%20lugar\%20seguro.pdf

Castro, Alejandro. (2009). Violencia silenciosa en la escuela. Dinámica del acoso escolar y laboral. Buenos Aires, Argentina: Bonum.

Comellas, María Jesús y Lojo, Mirta. (2009). Un cambio de mirada para abordar y prevenir la violencia en la escuela. El grupo como posibilidad de socialización y de convivencia. España: Octaedro.

Cerezo, Fuensanta. (2007). La violencia en las aulas. Madrid, España: Pirámide

Chagas, Raquel. (2005). Los maestros frente a la violencia entre alumnos. Revista Mexicana de Investigación Educativa, 10(27) 1071-1082.

Fondo de las Naciones Unidas para la Infancia (Unicef). (2011). Por qué, cuándo y cómo intervenir desde la escuela ante el maltrato a la infancia y la adolescencia. Argentina: UNICEF. 
Hernández, Roberto, Fernández, Carlos y Baptista, Pilar. (2007). Metodología de la investigación (4⿳亠丷a . ed.). México: Mc Graw Hill.

Machado, Jesús y Guerra, José Gregorio. (2009). Violencia en la escuela. Recuperado de http://www.gumilla.org/biblioteca/bases/biblo/texto/SIC2009715 211-218.pdf

Martínez-Otero, Valentín. (2005). Conflictividad escolar y fomento de la convivencia. Revista Iberoamericana de Educación, (38), 89-107.

Torrego, Juan Carlos. (2006). Modelo integrado de mejora de la convivencia. Estrategias de mediación y tratamiento de conflictos. Barcelona: Graó.

Tomasini, Marina, Domínguez, Lucía y Peralta, Heliana. (2013). La violencia entre estudiantes desde el punto de vista de sus actores. Una narrativa de la sociabilidad juvenil. Revista Ciencias Sociales. (58), 1-45. Recuperado de http://www.apostadigital.com/revistav3/hemeroteca/tomasini.pdf

Valadez, Isabel. (2008). Violencia escolar: maltrato entre iguales en escuelas secundarias de la zona metropolitana de Guadalajara. México: Mar-Eva. Recuperado de http://cvsp.cucs.udg.mx/drupal6/documentos/violencia escolar libro.pdf 